\title{
Breakdown of the Nagaoka phase at finite doping
}

\author{
Ilya Ivantsov, ${ }^{1,2}$ Alvaro Ferraz, ${ }^{3}$ and Evgenii Kochetov ${ }^{1}$ \\ ${ }^{1}$ Bogoliubov Laboratory of Theoretical Physics, Joint Institute for Nuclear Research, Dubna, Russia \\ ${ }^{2}$ L. V. Kyrensky Institute of Physics, Siberian Branch of Russian Academy of Sciences, Krasnoyarsk, Russia \\ ${ }^{3}$ International Institute of Physics - UFRN, Department of Experimental and Theoretical Physics - UFRN, Natal, Brazil
}

(Received 16 December 2016; revised manuscript received 27 March 2017; published 11 April 2017)

\begin{abstract}
The Nagaoka $(U=\infty)$ limit of the Hubbard model on a square lattice is mapped onto the itinerant-localized Kondo model at infinitely strong coupling. Such a model is well suited to perform quantum Monte Carlo (QMC) simulations to compute spin correlation functions. For periodic boundary conditions, this model is shown to exhibit no short-range ferromagnetic (FM) spin correlations at any doping $\delta \geqslant 0.01$ and at finite temperature $T=0.1 t$. Our simulations give no indication that there is a tendency towards ferromagnetic ordering in the ground state, with more than one hole. Employing on the other hand the open boundary conditions (or mixed boundary conditions) may result in the qualitatively different results for the thermodynamic limit depending on the way one chooses to approach this limit. These observations imply that the relevant thermodynamic limit remains unclear.
\end{abstract}

DOI: 10.1103/PhysRevB.95.155115

\section{INTRODUCTION}

The strong electron correlations are at work to full extent in the Nagaoka $(U=\infty)$ limit of the Hubbard model. Indeed, in this case an infinitely strong Coulomb repulsion strictly prohibits the double electron occupancy of the lattice sites, and the no double occupancy (NDO) constraint becomes of the utmost importance. In the infinite $U$ limit, the Hubbard Hamiltonian reduces to

$$
H_{U=\infty}=-\sum_{i j, \sigma} t_{i j} \tilde{c}_{i \sigma}^{\dagger} \tilde{c}_{j \sigma}, \quad \tilde{c}_{i \sigma}^{\dagger}=c_{i \sigma}^{\dagger}\left(1-n_{i-\sigma}\right),
$$

where $t_{i j}$ is a symmetric matrix whose elements represent the hopping amplitude $t>0$ between the nearest-neighbor sites and which are, otherwise, zero. Despite its seemingly simple form, this Hamiltonian cannot be diagonalized due to the fact that the projected electron operators $\tilde{c}_{\sigma}$ fulfill complicated commutation relations resulting from the explicit manifestation of strong correlations.

The physics behind the model (1) is certainly far from trivial. Indeed, Nagaoka [1] proved a theorem stating that for one hole the ground state of the $U=\infty$ Hubbard model is a fully saturated ferromagnet. This provides an interesting example of a quantum system in which ferromagnetism appears as a purely kinetic energy effect with hole hopping (itinerant ferromagnetism) emerging as a result of the strong correlations from the NDO constraint.

Unfortunately, despite extensive work over many years, this model and itinerant ferromagnetism are still poorly understood. One of the important questions to be addressed concerns the thermodynamic stability of the Nagaoka phase. That is, whether or not the Nagaoka state is stable when the density of holes is finite in the thermodynamic limit. There are arguments both for [2-9] and against [10-13] the thermodynamic stability of the Nagaoka phase and comparisons between various approaches have been made carefully (for a recent example, see [14]). The basic problem that prevents one from reaching a definite conclusion on that is the large- $U$ limit or, equivalently, the local NDO constraint which is very difficult to deal with in a controlled way.
Analytical approaches basically imply a mean-field (MF) treatment in which the local NDO constraint is uncontrollably replaced by a global condition. For example, the standard slave fermion (SF) MF theory which treats the NDO constraint only on average is known to predict a stable FM phase for the $U=\infty$ Hubbard model over an unphysically large doping range. It was however shown that the SF MF approach produces spurious results and it is therefore not reliable for the description of the Nagaoka ferromagnetism [15].

Available variational approaches [2,4,8,16-19] show that variational estimations that involve more realistic refined trial wave functions result in a smaller value of the critical hole concentration. For example, by advanced analytical means, a rather small upper bound on the critical hole concentration, namely $\delta_{c}=0.25$, was obtained [20]. This result has been recently confirmed by the variational Monte Carlo investigation [21]. In case the mean-field treatment provides an exact result, i.e., in infinite spatial dimensions, the fully polarized FM ground state is never stable [22]. One might therefore think that a proper treatment of the NDO constraint could improve the MF results shifting the critical hole concentrations towards progressively smaller values.

\section{MODEL}

In the present section we reformulate the standard infinite $U$-Hubbard model (1) in a form that explicitly takes into account some basic facts concerning the physics of strongly correlated electrons at low doping. This enables us to apply numerical quantum Monte Carlo calculations in a more efficient way.

In the underdoped cuprates, one striking feature is the simultaneous localized and itinerant nature of the lattice electrons. One might hope therefore that representing the model (1) in a form that takes both aspects into consideration on equal footing would help to address the problem in a more efficient way. To this end, Ribeiro and Wen proposed a slave-particle spin-dopon representation of the projected electron operators in the enlarged Hilbert space [23],

$$
\tilde{c}_{i}^{\dagger}=c_{i}^{\dagger}\left(1-n_{i-\sigma}\right)=\frac{1}{\sqrt{2}}\left(\frac{1}{2}-2 \vec{S}_{i} \vec{\sigma}\right) \tilde{d}_{i} .
$$


In this framework, the localized electron is represented by the lattice spin $\vec{S} \in s u(2)$, whereas the doped hole (dopon) is described by the projected hole operator $\tilde{d}_{i \alpha}=d_{i \sigma}\left(1-n_{i-\alpha}^{d}\right)$. Here $\tilde{c}^{\dagger}=\left(\tilde{c}_{\uparrow}^{\dagger}, \tilde{c}_{\downarrow}^{\dagger}\right)^{t}$ and $\tilde{d}=\left(\tilde{d}_{\uparrow}, \tilde{d}_{\downarrow}\right)^{t}$.

In terms of the projected electron operators, the constraint of no double occupancy (NDO) that encodes the essence of strong correlation takes on the form

$$
\sum_{\alpha}\left(\tilde{c}_{i \alpha}^{\dagger} \tilde{c}_{i \alpha}\right)+\tilde{c}_{i \alpha} \tilde{c}_{i \alpha}^{\dagger}=1 .
$$

It singles out the physical 3D on-site Hilbert space. Only under this condition the projected electron operators are isomorphic to the Hubbard operators. Within the spin-dopon representation (2), the NDO constraint reduces to a Kondotype interaction [24]

$$
\vec{S}_{i} \cdot \vec{s}_{i}+\frac{3}{4}\left(\tilde{d}_{i \uparrow}^{\dagger} \tilde{d}_{i \uparrow}+\tilde{d}_{i \downarrow}^{\dagger} \tilde{d}_{i \downarrow}\right)=0,
$$

with $\vec{s}_{i}=\sum_{\alpha, \beta} \tilde{d}_{i \alpha}^{\dagger} \vec{\sigma}_{\alpha \beta} \tilde{d}_{i \beta}$ being the dopon spin operator.

The on-site operator

$$
\mathcal{P}=1-\vec{S}_{i} \cdot \vec{s}_{i}+\frac{3}{4}\left(\tilde{d}_{i \uparrow}^{\dagger} \tilde{d}_{i \uparrow}+\tilde{d}_{i \downarrow}^{\dagger} \tilde{d}_{i \downarrow}\right), \quad \mathcal{P}^{2}=\mathcal{P}
$$

commutes with the Hamiltonian and projects out the unphysical states. In terms of the projectors, Eqs. (2) can be rewritten in the form

$$
\tilde{c}_{i \sigma}^{\dagger}=\sqrt{2} \operatorname{sgn}(\sigma) \mathcal{P}_{i} \tilde{d}_{i-\sigma} \mathcal{P}_{i},
$$

where $\operatorname{sgn}(\sigma=\uparrow, \downarrow)= \pm$. In view of this, we have

$$
H_{U=\infty}=\mathcal{P} \sum_{i j, \sigma} 2 t_{i j} \tilde{d}_{i \sigma}^{\dagger} \tilde{d}_{j \sigma} \mathcal{P}, \quad \mathcal{P}=\prod_{i} \mathcal{P}_{i} .
$$

Equivalently, Eq. (6) can be represented in the form of the lattice Kondo model at dominantly strong Kondo coupling $\lambda \gg t[24]$ :

$$
H_{U=\infty}=\sum_{i j \sigma} 2 t_{i j} d_{i \sigma}^{\dagger} d_{j \sigma}+\lambda \sum_{i}\left(\vec{S}_{i} \cdot \vec{s}_{i}+\frac{3}{4} n_{i}^{d}\right),
$$

where we have dropped the "tilde" sign of the dopon operators, as it becomes irrelevant in the presence of the NDO constraint. In spite of the global character of the parameter $\lambda$, it enforces the NDO constraint locally due to the fact that the on-site physical Hilbert subspace corresponds to zero eigenvalues of the constraint, whereas the nonphysical subspace is spanned by the eigenvectors with strictly positive eigenvalues.

The unphysical doubly occupied electron states are separated from the physical sector by an energy gap $\sim \lambda$. In the $\lambda \rightarrow+\infty$ limit, i.e., in the limit in which $\lambda$ is much larger than any other existing energy scale in the problem, those states are automatically excluded from the Hilbert space. Right at the point $\lambda=+\infty$ the proposed model (7) is equivalent to the $(U=\infty)$ Hubbard model of strongly correlated electrons. Away from that point, this model describes a phenomenological Kondo model in which the strength of the correlation is controlled by $\lambda$. In $1 \mathrm{D}, \mathrm{Eq}$. (7) reproduces the well-known exact results for the $(U=\infty)$ Hubbard model [25].

At this point a following remark is in order, concerning the correspondence between the two limits $U \rightarrow \infty$ and $\lambda \rightarrow \infty$. It might seem that this limit is equivalent to merely setting $t_{i j}=0$ in Eq. (7) which reduces the problem to the on-site one. This is however not true for the original Hubbard model, where the eigenfunctions are strongly entangled and very complicated.

The point is that, in the strong coupling limit $(\lambda / t \gg 1)$, the local spin-spin correlator between conduction dopons and localized moments $\left\langle\vec{S}_{i} \cdot \vec{s}_{i}\right\rangle$ converges to a value of $-3 / 4\left\langle n_{d}\right\rangle$. This is precisely canceled out by the term $3 / 4 n_{d}$ that enters the kinetic term in Eq. (7). Because of this the theory remains finite. The energy per site takes on the form

$$
E=A+O\left(\lambda^{-1}\right) \text {. }
$$

It starts with a $\lambda$ independent term $A$ since all the unphysical states are excluded in this strong coupling limit. The leading term depends on $t$ in a nontrivial way, since in the large $\lambda$ limit the underlying Hilbert space is modified by the NDO constraint. For example, in 1D, the leading term takes the form [25]

$$
A=-\frac{2 t}{\pi} \sin (\pi \delta),
$$

where $\delta$ is a hole concentration. The corresponding subleading terms can be found using the results for a strongly coupled 1D Kondo model [26].

\section{EXACT DIAGONALIZATION: PERIODIC BOUNDARY CONDITIONS}

\section{A. Large clusters with one and two holes}

To get some insight into what kind of magnetic order one might expect at finite doping, we start by performing exact diagonalization of the finite lattice clusters. In this as well as in the two subsequent sections, we use periodic boundary conditions (PBCs). Some interesting and important modifications caused by the use of other possible boundary conditions will be discussed in detail in Sec. V.

The size of the Hamiltonian matrix to be diagonalized is $3^{N} \times 3^{N}$, where $N$ is the number of sites. This makes a diagonalization difficult even for $N \sim 20$. However, the Hamiltonian is a block-diagonal matrix, where each block corresponds to a given number of electrons and a total spin projection. Let us first restrict a number of holes to that of $n_{h}=1,2$ and fix the total spin projection to be $Q_{z}=$ $Q_{\max }, Q_{\max }-1$. In this case we can significantly enlarge the lattice size due to the fact that the size of the largest Hamiltonian block $\left(n_{h}=2, Q=Q_{\max }-1\right)$ is proportional to the $N(N-1)(N-2) / 2$.

In Fig. 1 we report the results on the exact diagonalization of finite clusters with a maximal size up to $12 \times 12$ sites and the periodical boundary conditions (PBCs). The upper curve corresponds to the case of $n_{h}=1$ and it displays the difference between a fully polarized state and a state with one spin flipped, $\Delta E=E\left(Q_{\max }-1\right)-E\left(Q_{\max }\right)$. In case of one hole our results agree with Nagaoka's theorem, which predicts that a fully polarized state is the ground state. The lower curve displays the same quantity for the case of $n_{h}=2$. In case of two holes the energy of a fully polarized state lies higher than that of a state one spin flipped for all the considered lattices sizes. Such a behavior of the energy levels shows that the fully polarized state is not a ground state for two holes. In other words, the Nagaoka state with two holes is unstable which fully agrees with results published elsewhere [27]. 


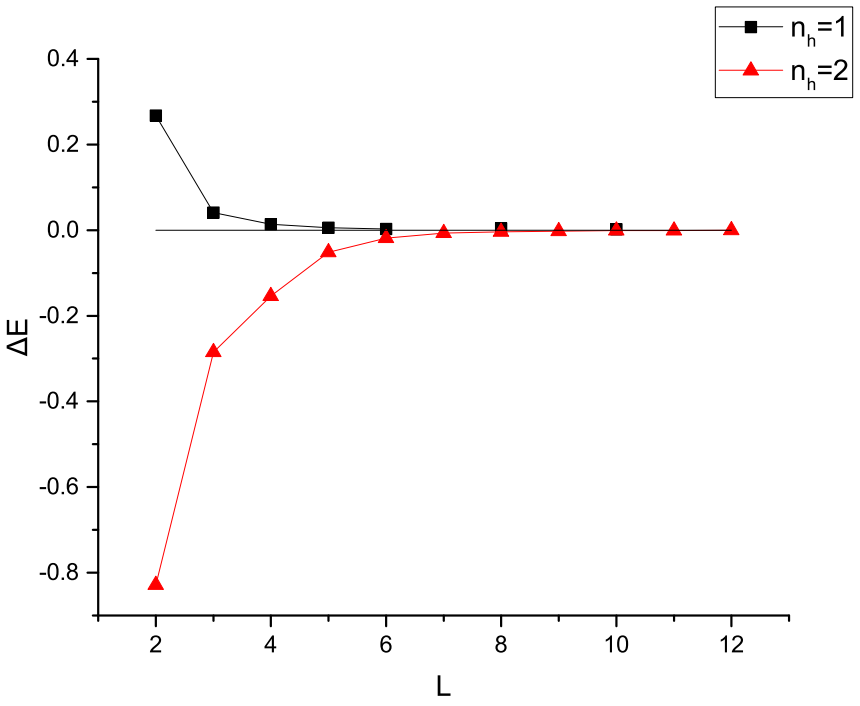

FIG. 1. The figure shows the energy difference $\Delta E=E\left(Q_{\max }-\right.$ 1) $-E\left(Q_{\max }\right)$ between the states with $Q=Q_{\max }$ and $Q=Q_{\max }-1$ for the different numbers of holes $n_{h}$. The lattice size $N=L \times L$ and the doping level $\delta=n_{h} / L^{2}$.

\section{B. Small cluster exact diagonalization}

We further proceed by computing the lowest energy as a function of various hole numbers $n_{h}$ and total spin projections $Q_{\text {ground }}$ for different clusters with the size up to $4 \times 4$. The obtained results presented in Fig. 2 show that a fully polarized FM state is a ground state only if there is one hole in a lattice. For more than one hole, a fully polarized state is never a ground state. Our results coincide exactly with those obtained by other methods [28].

On the other hand, for hard-core spinful bosons, the ground state appears as a fully polarized state at any number of holes. This agrees [29] with the statement exactly proven elsewhere that, for spinful bosons, the hard-core ferromagnetism is stable for all hole densities [30].

Another interesting issue to address concerns the character of a possible associated quantum phase transition. Let us assume that there is a thermodynamically stable fully polarized state at finite doping in the region of $0 \leqslant \delta \leqslant \delta_{\mathrm{cr}}$. Is then the onset of leading instability of this fully polarized phase implies that it occurs gradually, through small $\Delta Q=1$ changes in the total spin? Or instead, such a transition is discontinuous, meaning a large abrupt change in the total spin $\Delta Q \gg 1$ ? The results of the exact diagonalization of the small clusters displayed in Fig. 2 indicate that the transition from the Nagaoka state to the state with two holes always occurs through an abrupt spin change that increases with the lattice size. In particular, the results presented in Fig. 2(a) tell us that $\Delta Q=2$, whereas those for Fig. 2(d) indicate that $\Delta Q=7$. This therefore seems to indicate at first sight that the breakdown of the Nagaoka phase at $T=0$ is of a discontinuous character. However, the "oscillation" character of the curves displayed in Fig. 2 show that, at larger number of holes, $n_{h}>2$, there are in fact transitions with $\Delta Q=1$ or even $\Delta Q=0$. It is not therefore clear what type of transitions actually survives in the thermodynamic limit. In any event, the finite cluster calculations displayed in Fig. 2 do not support the statement that the destruction of the Nagaoka state is necessarily accompanied by an abrupt change of the total spin.

The exact small-cluster diagonalization can also be used to compute the spin-spin correlations $\left\langle Q_{i}^{z} Q_{i+1}^{z}\right\rangle$ between nearestneighbor sites. Figure 3 shows these correlations computed on the same clusters as those depicted in Fig. 2. In the hard-core boson case, the correlation functions are purely ferromagnetic and scale as $n_{e}^{2}$. This clearly corresponds to the fully polarized states. For the hard-core fermions, however, the correlations do not reveal any FM magnetic order except for the one-hole case, where the correlations coincide with those in the hard-core boson case. In the fermionic case, a sort of AFM order builds up instead, with a magnitude of the correlations decreasing with the total lattice size. In the next section, we confirm this result by QMC calculations for larger lattice clusters.

\section{QUANTUM MONTE CARLO CALCULATIONS}

An alternative approach is based on a computation of spinspin correlation functions by employing the QMC algorithm. In this way one can estimate a magnetic correlation length in the asymptotic regime $r \gg a$. In case this quantity shows no appreciable dependence on finite-size effects, one may draw certain reliable conclusions on a character of the underlying magnetic order.

One must however take proper care of the fact that we are dealing with a strong-coupling problem. Namely, since we are interested in the large $\lambda$ limit, it seems appropriate to separate the Hamiltonian (7) in the following way: $H_{U=\infty}=H_{0}+H_{\text {int }}$, where the leading term is now

$$
H_{0}=\lambda \sum_{i}\left(\vec{S}_{i} \cdot \vec{s}_{i}+\frac{3}{4} n_{i}^{d}\right),
$$

and the "interaction" term is

$$
H_{\mathrm{int}}=2 t \sum_{i j \sigma} d_{i \sigma}^{\dagger} d_{j \sigma}, \quad t / \lambda \ll 1 .
$$

Although the exact diagonalization remains of the same complexity, the spin-spin correlators can be computed in a more efficient way by employing the localized and itinerant degrees of freedom displayed by our model (7). The convergence of the QMC depends on a selected basis. Taking the Hamiltonian in this form allows us to achieve the significant weakening of the sign problem in the low-doped case due to two facts. First, it allows us to diagonalize the $H_{0}$ term in the one-site representation and rewrite $H_{\text {int }}$ in the new basis. During the simulation we can set $\lambda$ finite and then unphysical configurations that involve $\lambda$ terms can occasionally occur. Since all measurements occur in the absence of these configurations, they do not contribute to the final result. This approach significantly improves the ergodicity of the algorithm and does not contradict the detailed balance principle. Second, in those circumstances the sign problem weakens because of the fact that, in the spin-dopon representation, the greater the density of dopons, the smaller is the average sign. For example, in case of exactly one dopon, the sign problem is absent. However, if standard electron operators are used the average sign in the same case is extremely small, and this is something we must definitely avoid. 


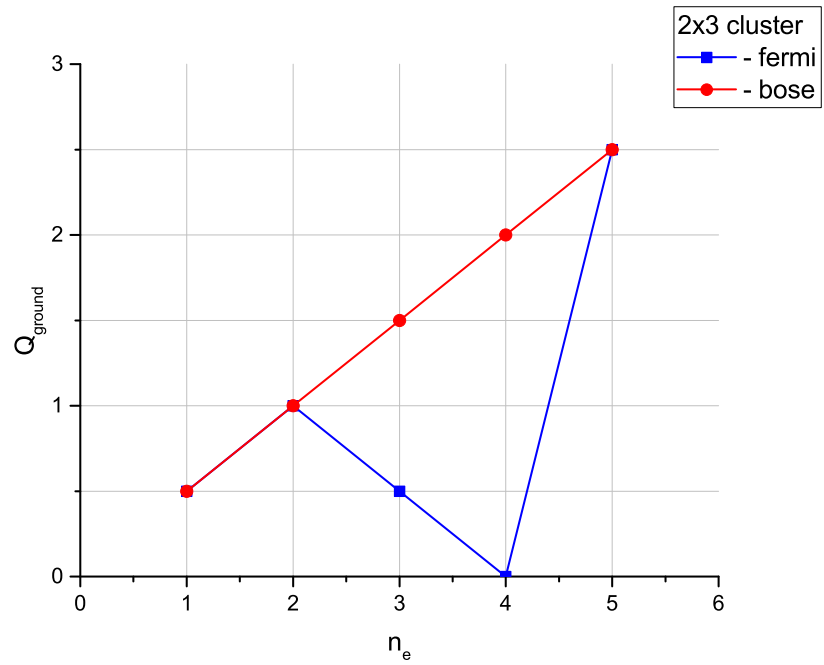

(a)

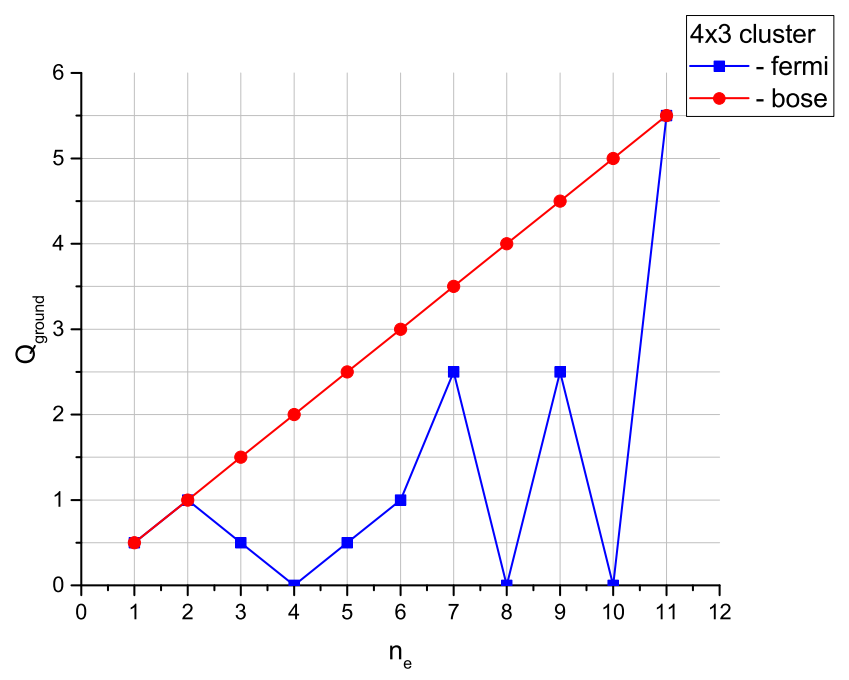

(c)

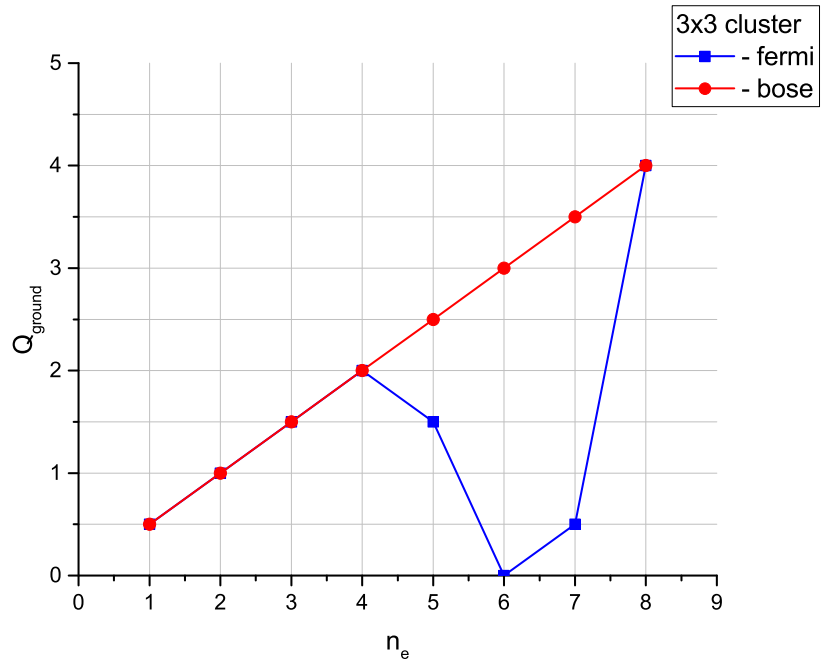

(b)

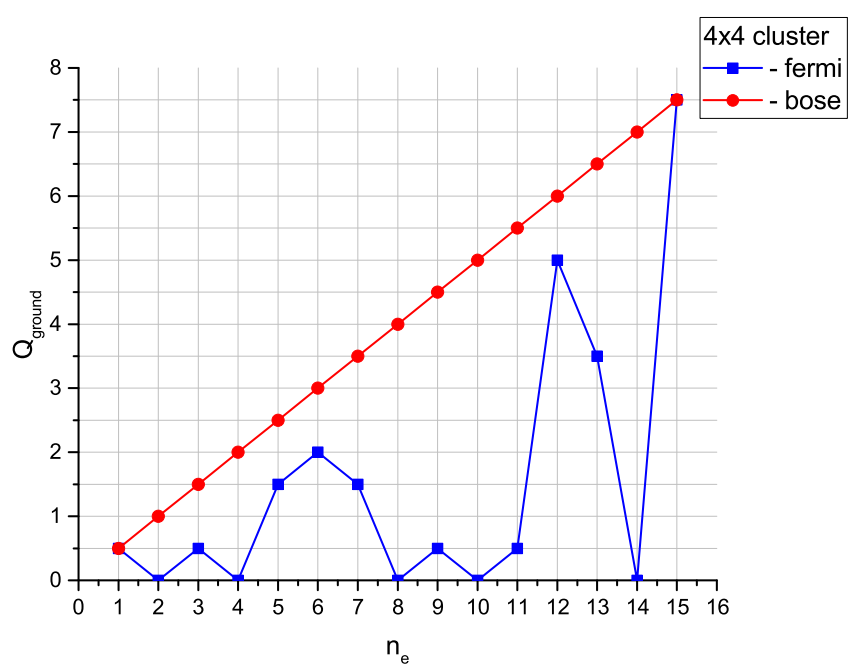

(d)

FIG. 2. The spin value in the ground state for the different numbers of electrons $n_{e}=N-n_{h}$. Periodic boundary conditions are imposed.

To compute the spin correlators we adopt the continuous time worldline (CTWL) QMC algorithm based on the representation of the partition function in the interaction picture:

$$
e^{-\beta H_{U=\infty}}=e^{-\beta H_{0}} T_{\tau}\left[\exp \left(-\int_{0}^{\beta} H_{\text {int }}(\tau) d \tau\right)\right],
$$

where $T_{\tau}$ stands for the $\tau$-ordering operator, and the partition function $Z_{U=\infty}=\operatorname{tr} e^{-\beta H_{U=\infty}}$. All the necessary details for the CTWL QMC algorithm to be applied to treat the lattice Kondo-Heisenberg model can be found in a recent paper published elsewhere [31]. The numerical simulations exposed in that work are simply to be restricted to the case of $J=0$.

The spin-spin correlation function $g(r)$ for the physical electron operators is calculated for a fixed number of dopons $\delta$ :

$$
g(r)=\frac{4}{\Delta(r)} \sum_{i j}\left\langle\left(S_{i}^{z}+s_{i}^{z}\right)\left(S_{j}^{z}+s_{j}^{z}\right)\right\rangle \bar{\delta}\left(r-\left|\mathbf{R}_{i}-\mathbf{R}_{j}\right|\right),
$$

where $\mathbf{R}_{i}$ is the radius vector of the site $i, \Delta(r)=\sum_{i j} \bar{\delta}(r-$ $\left.\left|\mathbf{R}_{i}-\mathbf{R}_{j}\right|\right)$, and

$$
\bar{\delta}(x)= \begin{cases}1, & \text { if }|x| \leqslant 0.5 a, \\ 0, & \text { otherwise, }\end{cases}
$$

with $a$ being the lattice constant and $\langle\cdots\rangle$ being an average over the spin configurations generated in the QMC run. The correlation function is normalized by the condition $g(r=0$; $\delta=0)=1$. All numerical results are obtained for a $20 \times 20$ lattice cluster with periodic boundary conditions.

Figure 4 displays the corresponding spin-spin correlators for the hard-core bosons [Fig. 4(a)] and the hard-core fermions [Figs. 4(b) and 4(c)]. In the boson case, the FM order is clearly observed in full accordance with the exact result [29]. The small-cluster exact diagonalization results displayed in Fig. 3 clearly show that the correlation function scales at $T=0$ as $n_{e}^{2}$. This implies that $g(r) \approx 1$ at $\delta \ll 1$. Finite-temperature effects considerably suppress the correlation function as seen in Fig. 4. However, it remains finite since $g(r) \approx 0.2, r \gg a$. 


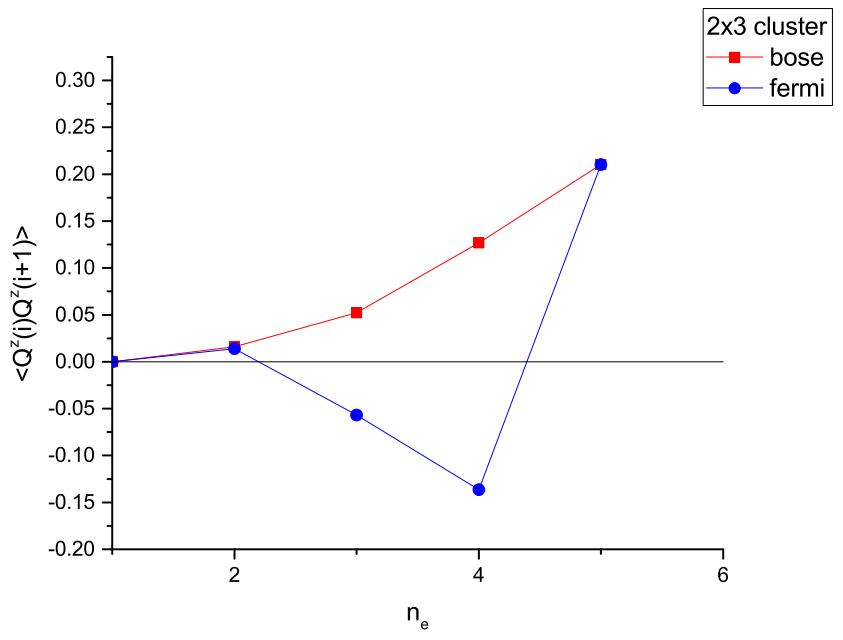

(a)

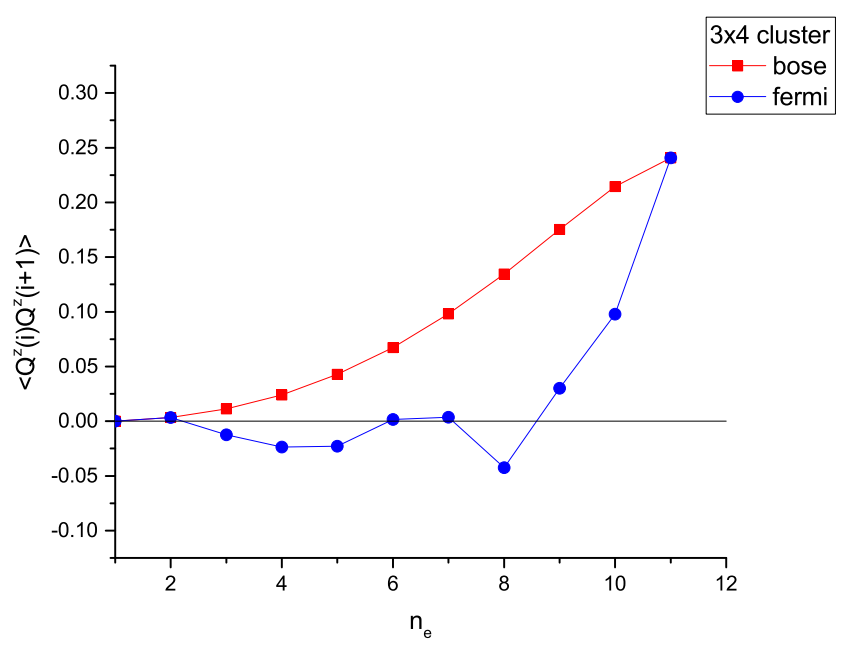

(c)

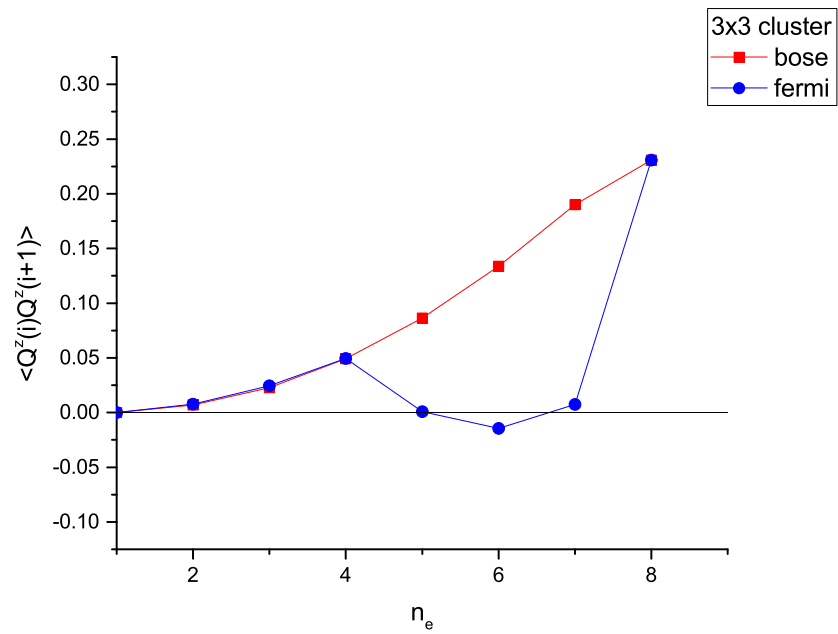

(b)

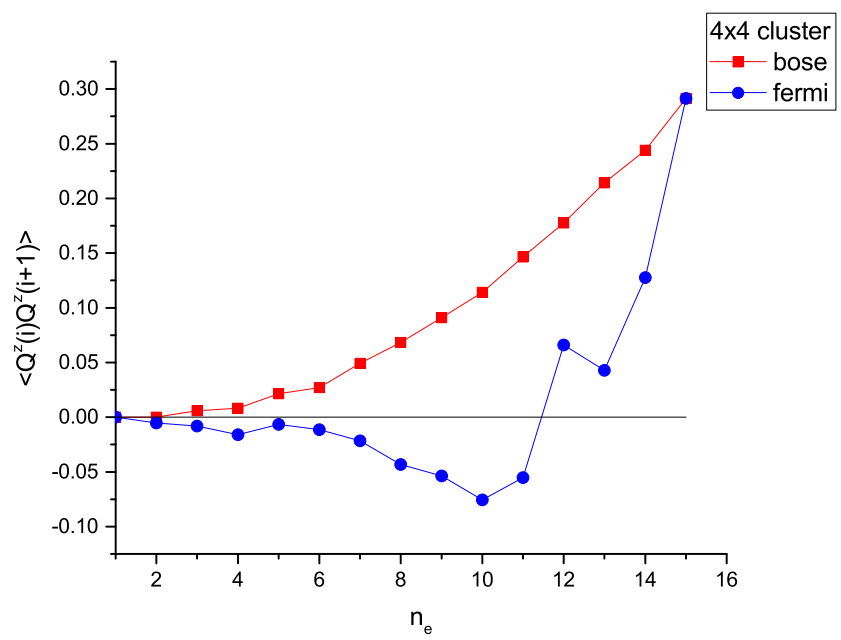

(d)

FIG. 3. The $\left\langle Q_{i}^{z} Q_{i+1}^{z}\right\rangle$ correlation between nearest sites for the different numbers of electrons $n_{e}=N-n_{h}$. Periodic boundary conditions are imposed.

The observed order in hard-core bosons case is of course not truly long ranged since it does not exist in $2 \mathrm{D}$ at finite temperature but rather quasi-long-range feature. The associated FM correlation length is finite but much larger than the cluster size. In fact it scales exponentially with $1 / T$ as $T \ll t$.

Figure 4(b) displays the fermion spin correlators for the different doping levels. In sharp contrast to the hard-boson case, there is no tendency towards FM ordering at finite doping. The spin-spin correlations in real space show no evidence of even short-range FM correlations, but rather weak $\mathrm{AF}$ correlations instead [32]. In case there indeed were a continuous phase transition at a critical doping $\delta_{c}$ at which a true long-range FM order in a ground state in 2D does emerge, then, in the associated quantum critical region specified in particular by the requirements $\delta=\delta_{c}, T \ll t$, the system must necessarily display a finite FM spin-spin correlation length that scales as $T^{-1 / z}$ where $z \geqslant 1$ is a dynamic exponent. This exponent parametrizes the relative scaling of space and time.
The precise value of $z$ could have been guessed provided an appropriate effective action to describe the low-energy physics of the $U=\infty$ Hubbard model would have been available, which however is not the case. Just to get an idea as to what might be the order of magnitude of the spin-spin correlation length away from the critical point, let us assume that $z=1$ (In Fermi-liquid-like systems this would imply that there are no overdamped modes associated with an ordering field [34]). In this case $\xi_{\mathrm{FM}} \propto \lambda_{T}$, where the de Broglie wavelength at finite temperature $\lambda_{T}=v / T$. This relation implies that thermal and quantum fluctuations are equally important in this (critical) regime. The characteristic velocity of the quasiparticle lowenergy excitations $v \propto t a$. At a given temperature $T=0.1 t$, we therefore get $\xi_{\mathrm{FM}} \propto 10 \mathrm{a}$. In other words, the long-range magnetic order in the ground state would manifest itself at finite temperature through a finite correlation length of order of at least a few lattice spacings. We however never observe the spin-spin correlation functions that display such a behavior down to a very small doping level of $\delta=0.01$. 


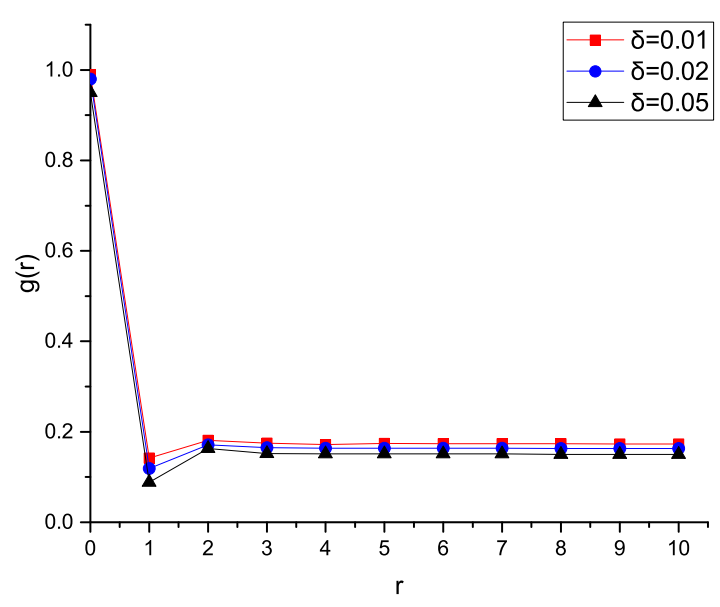

(a)

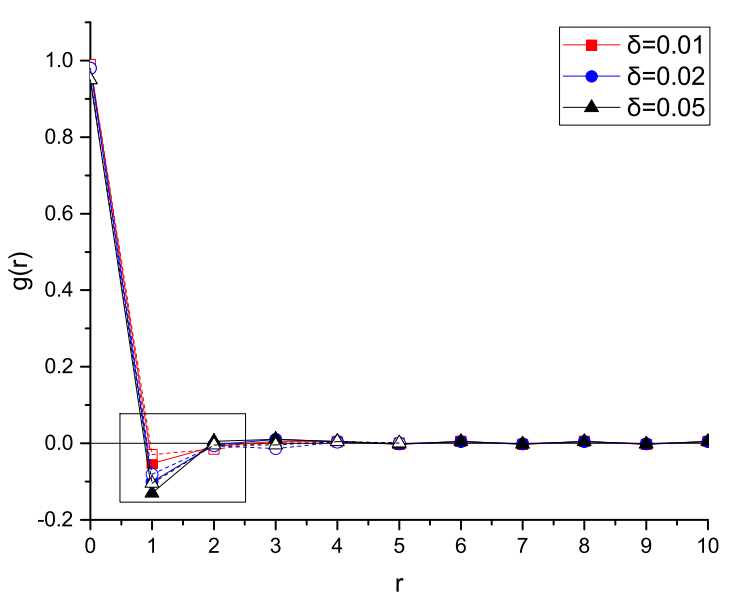

(b)

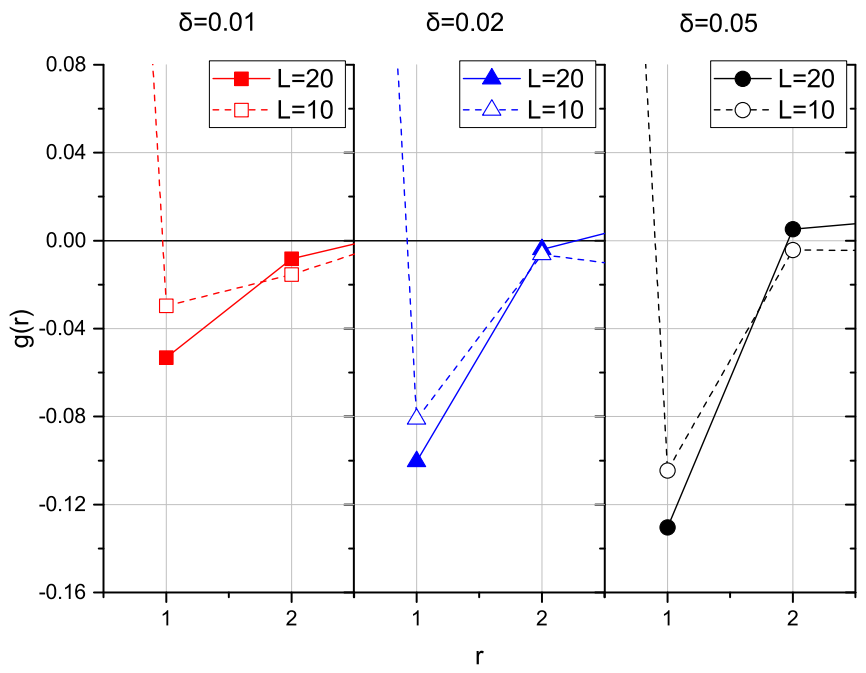

(c)

FIG. 4. (a) Correlation functions for $L=10$ for different doping levels for the hard-core boson case at $T=0.1 t$. (b) The same for fermion case. Solid (dashed) lines show results obtained for $L=20$ ( $L=10$ ), respectively. Number of lattice size is $N=L \times L$. (c) The highlighted fragments of (b) with the doping level being separately presented.

\section{DISCUSSION: ROLE OF BOUNDARY CONDITIONS}

We have shown that the spin-spin correlation function analysis provides strong arguments against thermodynamic stability of the Nagaoka phase. However, a different conclusion was reached in a recent work [35] that employs a density matrix renormalization group (DMRG) approach to study a phase diagram of the infinite $U$ Hubbard model on two- to six-leg ladders. The authors find a fully polarized FM phase at zero temperature, when $\delta$, the density of holes per site, is in the range $0<\delta<0.2$. As those results are largely insensitive of the ladder width, they consider them representative of the 2D square lattice. These two conclusions seemingly contradict each other. In this section we intend to clarify this issue.

The important distinction between our work and the paper [35] has to do with the choice of boundary conditions. In the latter open boundary conditions (OBCs) are used, whereas we use the PBCs instead. We check by the exact diagonalization on rather large clusters how significant is this difference. To this end, we consider $12 \times 12$ clusters with one and two holes. As we showed in Sec. III, in this case, the PBCs imply that the two-hole Nagaoka (fully polarized) state is never a ground state of the system. In case of the OPB a one-hole state is again fully polarized as it should be as is in the case for the PBC in agreement with Nagaoka's theorem. However, the two-hole case is now represented by the two distinct phases.

Figure 5 shows the phase separation for the different lattice sizes and different boundary conditions, with Fig. 5(a) corresponding to the OBCs. The fully polarized (FP) phase implies that the $E\left(Q=Q_{\max }\right)<E\left(Q=Q_{\max }-1\right)$. In this case, the FP ground state is allowed though it is not necessarily realized. In contrast, the one spin flipped (SF) phase depicted by the shaded area corresponds to the case of $E(Q=$ $\left.Q_{\max }\right)>E\left(Q=Q_{\max }-1\right)$. This phase strictly prohibits a fully polarized ground state, because an energy of at least one spin flipped state is lower than that of the fully polarized state. The phase diagram in Fig. 5(a) is in an accordance with the results of Ref. [35]. At sufficiently large length of the two-, four-, six-leg chains a fully polarized two-hole state is lower than that with one spin flipped.

Our calculations agree with those obtained within the DMRG approach for large enough chain length. For example, at the critical hole concentration $\delta_{c}=0.2$, a two-leg chain exhibits a fully polarized ground state only when the chain length is equal to or larger than 5 . In this case, the critical number of the doped holes at which the FP state becomes more favorable should be equal to $(2 \times 5) \times(1-0.8)=2$, which agrees with the results depicted in Fig. 5(a).

We thus see that the OBCs allow for the existence of the fully polarized ground state at a sufficiently large lattice (a length along one of the axes $\geqslant 5$ ). There is however one exception. As seen from Fig. 5(a), the square $L \times L$ lattice clusters with two holes have at least one state, which has lower energy that the fully polarized one. The Nagaoka state is unstable in this case. This is an interesting observation, since usually just square lattice clusters are used in the actual carrying out the $2 \mathrm{D}$-limiting procedure.

Let us now turn to the case of the so-called mixed boundary conditions (MBCs). The results are reported in Fig. 5(b). The 


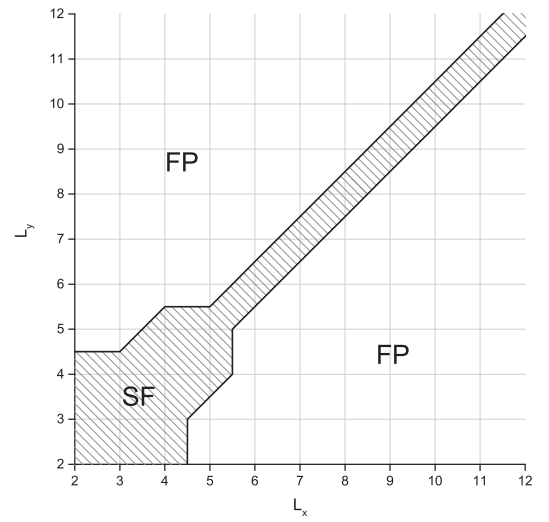

(a)

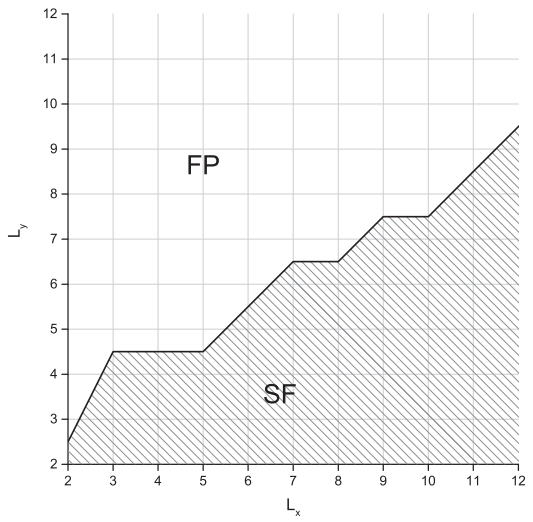

(b)

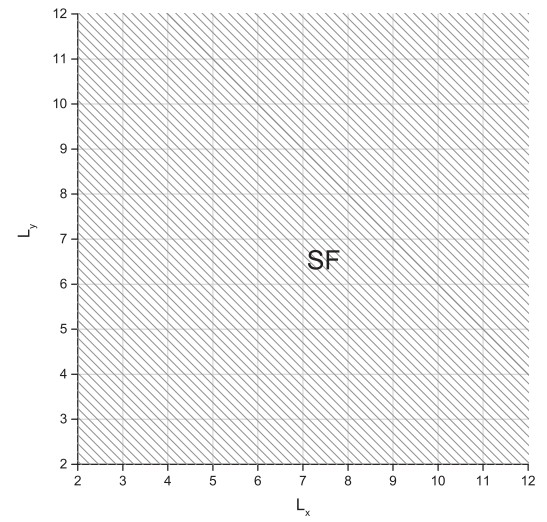

(c)

FIG. 5. The figure shows the energy difference between the fully polarized (FP) state $\left(Q=Q_{\max }\right)$ and the one spin flipped (SF) state $\left(Q=Q_{\max }-1\right)$ in case of two holes for the different lattice sizes $N=L_{x} \times L_{y}$. The SF areas correspond to the case $E\left(Q_{\max }-1\right)<E\left(Q_{\max }\right)$, the FP areas correspond to the case $E\left(Q_{\max }-1\right)>E\left(Q_{\max }\right)$. (a) The lattice with the open boundary conditions. (b) The lattice with the periodic boundary condition for the axis $x$ and the open boundary condition for the axis $y$. (c) The lattice with the periodic boundary conditions.

MBCs imply that the PBCs are imposed along the $x$ axis and the anti-PBCs along the $y$ axis. As seen from Fig. 5(b), in case the periodicity holds along the "long" side of a lattice rectangle, then the SF state is more favorable; in the opposite case, the fully polarized state is preferable. This is quite natural, since if a $2 \times 50$ lattice cluster is bent into a ring along the short side so that only two sites on the opposite sides interact, this clearly will produce no noticeable effects.

Finally, Fig. 5(c) shows that the PBCs used in our work imply that the state with $Q=Q_{\max }-1$ is always more favorable than the polarized one. In other words, the fully polarized state with the PBCs is never a ground state.

\section{CONCLUSION}

In conclusion, we show that there is an influence of the boundary conditions on the thermodynamic limit for the $(U=\infty)$ Hubbard model. The contribution that comes from the fixing boundary conditions may play a key role even for very large lattice cluster calculations. In principle, this happens because such contribution and that which comes from the difference in energy between a fully polarized and the unpolarized states are of the same order-O $O(1 / N)$ [27].

Within the approach based on the PBCs (for which the original Nagaoka theorem was formulated), the lack of a clear sign of FM short-range spin correlations in the parameter range studied in our work provides strong arguments against thermodynamic stability of the Nagaoka phase at least for the hole concentrations $\delta \geqslant 0.01$. It is very likely to conclude that the critical hole concentration is in fact equal to zero. There are actually only two acceptable options in the thermodynamic limit in this case, namely, either the FM order does not exist at all, which is the case for the hard-core fermions, or it is realized at all possible hole densities. This last (unphysical) option corresponds to implementing the hard-core bosonic statistics for the constituent particles. Employing on the other hand the OBCs (or MBCs) may result in the qualitatively different results for the thermodynamic limit depending on a way one chooses to approach this limit (by using square or rectangular building blocks, e.g., in case of the OBCs). These observations imply that the relevant thermodynamic limit remains unclear.

\section{APPENDIX}

In the Appendix we provide a derivation of the Nagaoka theorem within a framework of the spin-dopon representation. For simplicity we restrict ourselves to the case of a $D$ dimensional hypercubic regular lattice. In this case the sign of $t$ is irrelevant so that we can fix the Hamiltonian to be

$$
\begin{aligned}
H_{U=\infty}= & -2 t \sum_{i j \sigma} d_{i \sigma}^{\dagger} d_{j \sigma}+\frac{3 \lambda}{4} \sum_{i \sigma} d_{i \sigma}^{\dagger} d_{i \sigma} \\
& +\lambda \sum_{i} \vec{S}_{i} \cdot \vec{s}_{i}, \quad t>0 .
\end{aligned}
$$

The limit $\lambda \rightarrow \infty$ reduces the on-site Hilbert space to that comprising a spin-up state $|\uparrow\rangle_{i}=|\uparrow 0\rangle_{i}$, a spin-down state $|\uparrow\rangle_{i}=|\uparrow 0\rangle_{i}$, and a vacancy state $|0\rangle_{i}=\frac{|\uparrow \downarrow\rangle_{i}-|\downarrow \uparrow\rangle_{i}}{\sqrt{2}}$. We should therefore consider

$$
H=-2 t \sum_{i j \sigma} d_{i \sigma}^{\dagger} d_{j \sigma}
$$

in the reduced Hilbert space.

We define the basis one-vacancy states as

$$
|i,\{\sigma\}\rangle=\left|\sigma_{1} \sigma_{2} \cdots 0_{i} \cdots \sigma_{N}\right\rangle,
$$

where $\sigma_{k}=\uparrow \downarrow$ and $\{\sigma\}$ is a multi-index describing an arbitrary set of the lattice spins. The vacancy state $|0\rangle_{i}$ is a total spin singlet defined above.

Let

$$
|\Psi\rangle=\sum_{(i,\{\sigma\})} \psi_{(i, \sigma)}|i,\{\sigma\}\rangle
$$

be an arbitrary one-hole normalized state. Let us define a state $|\Phi\rangle$ with $Q=Q_{\max }=Q^{z}=(N-1) / 2$ as

$$
|\Phi\rangle=\sum_{i} \phi_{i}|i,\{\uparrow\}\rangle .
$$


Here $\vec{Q}=\sum_{i}\left(\vec{S}_{i}+\vec{s}_{i}\right)$ is a vector of the total spin of the electrons $\phi_{i}=\left(\sum_{\sigma}\left|\psi_{i, \sigma}\right|^{2}\right)^{1 / 2}$, and the multi-index $\{\uparrow\}$ represents that all the lattice spins point upwards. The energy of the state $|\Psi\rangle$ is evaluated to be

$$
\begin{aligned}
\langle\Psi|H| \Psi\rangle & =-t \sum_{i j, \sigma \tau} \bar{\psi}_{i \tau} \psi_{j, \sigma} \geqslant-t \sum_{i j} \bar{\phi}_{j} \phi_{i} \\
& \geqslant-t \sum_{i j}\left|\phi_{i}\right|^{2}=-t z,
\end{aligned}
$$

where $z$ is a coordination number. To obtain (A4) we have repeatedly used the Schwartz inequality [36]. From (A3) it follows that the energy of the ground state

$$
E_{\mathrm{gr}}=-t z \text {. }
$$

The last inequality in (A4) is saturated for the state (A3) provided $\phi_{i}=$ const $=1 / \sqrt{N}$. Such a state describes the fully polarized lattice spins and a hole with the highest mobility. This indicates that the fully polarized ferromagnetic state

$$
\left|\Phi_{\mathrm{gr}}\right\rangle=\frac{1}{\sqrt{N}} \sum_{i}|i,\{\uparrow\}\rangle
$$

is indeed the ground state of the system. This state has $Q=$ $Q^{z}=(N-1) / 2$.

Now it is left to show that there is no other state with $E=-t z$ and $Q<Q_{\max }$. Let us denote the state with $E=-t z$ and arbitrary given $N_{\uparrow}$ and $N_{\downarrow}$ by

$$
|\Phi\rangle=\sum_{(i\{\sigma\})} \psi(i \sigma)|i,\{\sigma\}\rangle .
$$

The Schrödinger equation

$$
H|\Phi\rangle=-t z|\Phi\rangle
$$

then gives

$$
\psi(i \sigma)=z^{-1} \sum_{j \tau=n[i \sigma]} \psi(j \tau) .
$$

Here $n[i \sigma]$ denotes nearest neighbors of $(i \sigma)$. The unique solution to (A5) reads [1]

$$
\psi(i \sigma)=\text { const. }
$$

This again corresponds to the state with $Q=Q_{\max }$ and $Q_{z}=$ $(N-1) / 2$, so that there is no state with $E=-t z$ and $Q<$ $Q_{\max }$.
[1] Y. Nagaoka, Phys. Rev. 147, 392 (1966).

[2] P. Richmond and G. Rickayzen, J. Phys. C 2, 528 (1969).

[3] J. A. Riera and A. P. Young, Phys. Rev. B 40, 5285 (1989).

[4] M. W. Long, in The Hubbard Model, Recent Results, edited by M. Rasetti (World Scientific, Singapore, 1991).

[5] H. Yokoyama and H. Shiba, J. Phys. Soc. Jpn. 56, 3570 (1987); D. Dzierzava and R. Frésard, Z. Phys. B 91, 245 (1993).

[6] E. Müller-Hartmann, Th. Hanisch, and R. Hirsh, Physica B 186188, 834 (1993).

[7] E. G. Goryachev and D. V. Kuznetsov, Pis'ma Zh. Eksp. Teor. Fiz. 56, 205 (1992) [JETP Lett. 56, 203 (1992)].

[8] F. Becca and S. Sorella, Phys. Rev. Lett. 86, 3396 (2001).

[9] A. Mielke and H. Tasaki, Commun. Math. Phys. 158, 341 (1993).

[10] A. Suto, Commun. Math. Phys. 140, 43 (1991).

[11] G. S. Tian, Phys. Rev. B 44, 4444 (1991); J. Phys. A 24, 513 (1991).

[12] W. O. Putikka, M. U. Luchini, and M. Ogata, Phys. Rev. Lett. 69, 2288 (1992).

[13] H. Tasaki, Prog. Theor. Phys. 99, 489 (1998).

[14] H. Park, K. Haule, C. A. Marianetti, and G. Kotliar, Phys. Rev. B 77, 035107 (2008).

[15] F. L. Braghin, A. Ferraz, and E. Kochetov, Phys. Rev. B 78, 115109 (2008).

[16] B. S. Shastry, H. R. Krishnamurthy, and P. W. Anderson, Phys. Rev. B 41, 2375 (1990).

[17] W. von der Linden and D. Edwards, J. Phys. Condens. Matter 3, 4941 (1991).

[18] A. G. Basile and V. Elser, Phys. Rev. B 41, 4842 (1990).

[19] Th. Hanisch, G. S. Uhrig, and E. Muller-Hartmann, Phys. Rev. B 56, 13960 (1997).
[20] P. Wurth, G. S. Uhrig, and E. Müller-Hartmann, Ann. Phys. (Leipzig) 5, 148 (1996).

[21] G. Carleo, S. Moroni, F. Becca, and S. Baroni, Phys. Rev. B 83, 060411(R) (2011).

[22] P. Fazekas, B. Menge, and E. Müller-Hartmann, Z. Phys. B 78, 69 (1990).

[23] T. C. Ribeiro and X.-G. Wen, Phys. Rev. Lett. 95, 057001 (2005); Phys. Rev. B 74, 155113 (2006).

[24] A. Ferraz, E. Kochetov, and B. Uchoa, Phys. Rev. Lett. 98 , 069701 (2007); R. T. Pepino, A. Ferraz, and E. Kochetov, Phys. Rev. B 77, 035130 (2008).

[25] A. Ferraz and E. Kochetov, Eur. Phys. J. B 86, 512 (2013).

[26] M. Sigrist, H. Tsunetsugu, K. Ueda, and T. M. Rice, Phys. Rev. B 46, 13838 (1992).

[27] B. Doucot and X. G. Wen, Phys. Rev. B 40, 2719 (1989).

[28] M. Takahashi, J. Phys. Soc. Jpn. 51, 3475 (1982).

[29] A. Fledderjohann, A. Langari, E. Müller-Hartmann, and K.-H. Mütter, Eur. Phys. J. B 43, 471 (2005).

[30] The hard-core boson model is given by Eq. (1) in which the hard-core fermion operators $\tilde{c}_{i \sigma}$ are replaced with the hard-core boson ones $b_{i \sigma}, \sum_{\sigma} b_{i \sigma}^{\dagger} b_{i \sigma} \leqslant 1$.

[31] I. Ivantsov, A. Ferraz, and E. Kochetov, Phys. Rev. B 94, 235118 (2016).

[32] Within the QMC simulations of the 2D Hubbard model, no indication of ferromagnetism was also found at $U=8 t, \delta=$ 0.35 , and $T=0.3 t$ on a $6 \times 6$ square lattice in [33].

[33] J. E. Hirsch, Phys. Rev. B 31, 4403 (1985).

[34] J. A. Hertz, Phys. Rev. B 14, 1165 (1976).

[35] L. Liu, H. Yao, E. Berg, S. R. White, and S. A. Kivelson, Phys. Rev. Lett. 108, 126406 (2012).

[36] H. Tasaki, Phys. Rev. B 40, 9192 (1989). 